Pacific Journal of Mathematic 


\section{MEAN PLAY OF SUMS OF POSITIONAL GAMES}

\section{OLOF HANNER}

1. Introduction. In 1953 Milnor studied certain positional 2-person games and defined what he called sums of such games [1]. He investigated the optimal strategies for these games and gave some information about them in terms of properties of the individual games.

In this paper we shall consider some other strategies for these sum games. They are in general not optimal. However, the difference between what a player gets when playing one of them instead of playing an optimal strategy can be estimated. For the sum of $n$ copies of the same game this difference is bounded for all $n$. Hence, in mean this difference is small for large $n$.

2. Description of the games. Essentially following Milnor [1] we describe the games as follows.

Each game contains a finite set of positions $P$. There are two players, $A_{1}$ and $A_{2}$. For each $p \in P$ and each player $A_{i}, i=1,2$, there is a set of possible moves $M_{i}(p) \subset P$. For each $p$ either both $M_{1}(p)$ and $M_{2}(p)$ contain at least one move or they are both vacuous. In the latter case $p$ is called an end position. For any chain $p_{0}, p_{1}, \cdots, p_{l}$ with $p_{j+1} \in M_{1}\left(p_{j}\right) \cup M_{2}\left(p_{j}\right)$, we shall have $p_{j} \neq p_{k}$ for $j \neq k$. The maximal number $l$ of steps in all such chains starting with $p_{0}=p$ will be denoted by $l(p)$. Then

$$
p_{1} \in M_{1}(p) \cup M_{2}(p) \text { implies } l\left(p_{1}\right)<l(p) .
$$

Note that a pass, $p \in M(p)$, is never possible. The positions with $l(p)=0$ are just the end positions.

For each end position the payoff functions $k_{1}(p)=-k_{2}(p)$ are defined. They shall satisfy a condition given below. The players start with some position and move alternatively until an end position is reached. Then each player collects his payoff.

For each player $A_{i}$ and position $p$, let $v_{i}(p)$ be the value of the game for $A_{i}$ when it is his turn to move at position $p$. It is given by

$$
\begin{array}{ll}
v_{i}(p)=k_{i}(p) & \text { for } l(p)=0, \\
v_{i}(p)=\max \left\{-v_{3-i}\left(p_{1}\right) \mid p_{1} \in M_{i}(p)\right\} & \text { for } l(p)>0 .
\end{array}
$$

Because of (2.1) these formulas define $v_{i}(p)$ by induction on $l(p)$.

The numbers $k_{i}(p)$ are defined when $p$ is an end position. We require that they shall be given in such a way that

Received August 12, 1958. 


$$
v_{1}(p)+v_{2}(p) \geqq 0
$$

Since the value at $p$ for $A_{i}$ is $v_{i}(p)$ if he has the move and $-v_{3-i}(p)$ if the other player has the move, the amount $v_{1}(p)+v_{2}(p)$ is the gain for a player of having the move. Inequality (2.3) therefore says that it is at least as good to move as to pass (if this would be allowed).

3. Sums of games. We now define the sum of two games $G$ and $G^{\prime}$. A position in the sum game $G+G^{\prime}$ is a pair $\left(p, p^{\prime}\right) \in P \times P^{\prime}$. A move in $G+G^{\prime}$ is a move in one of the games $G$ and $G^{\prime}$ and a pass in the other. Thus the moves in position $p+p^{\prime}=\left(p, p^{\prime}\right)$ are

$$
M_{i}\left(p+p^{\prime}\right)=M_{i}(p) \times p^{\prime} \cup p \times M_{i}\left(p^{\prime}\right) .
$$

We notice that

$$
l\left(p+p^{\prime}\right)=l(p)+l\left(p^{\prime}\right) .
$$

In particular the condition is still satisfied that in a chain of successive positions all positions are different. The position $p+p^{\prime}$ is an end position if and only if $p$ and $p^{\prime}$ both are end positions. For the end positions we define $k_{i}\left(p+p^{\prime}\right)$ by

$$
k_{i}\left(p+p^{\prime}\right)=k_{i}(p)+k_{i}\left(p^{\prime}\right) .
$$

It is not obvious that the sum of two games satisfying condition (2.3) also satisfies this condition. That this is the fact was proved by Milnor [1]. It will also be proved in $\S 8$ below as a consequence of Theorem 1.

It is clear that game addition is an associative and commutative operation and that the formulas corresponding to (3.1) and (3.2) hold for the sum of any finite number of games. A move in the sum of several games is a move in one of them and a pass in all the others.

4. The main problem. The problem for us will be to give good strategies for sums of games in terms of properties of the individual games. Then we must decide what kind of strategies we shall consider to be good.

One way to attack this problem is as follows. Consider $n$ copies of a game $G$ and take their sum $n G$. Let them all be started in the same position $p$. Then the value of the sum game is $v_{i}(n p)$, where we have written $n p$ instead of $p+\cdots+p$. Now, what happens to the mean value $v_{i}(n p) / n$ when $n$ tends to infinity? In fact this number tends to a limit $m_{i}(p)$ which will be called the mean value of the game $G$ at $p$. In later sections we shall prove that $m_{i}(p)$ satisfies

$$
m_{1}(p)+m_{2}(p)=0,
$$




$$
m_{i}(p) \leqq v_{i}(p)
$$

If we change $i$ to $3-i$ in (4.2) and apply (4.1) we get

$$
-v_{3-i}(p) \leqq m_{i}(p) \text {. }
$$

Thus $m_{i}(p)$ lies between $v_{i}(p)$ and $-v_{3-i}(p)$ which represent the values for $A_{i}$ when the game is started at $p$ by him or by $A_{3-i}$ respectively.

Of a good strategy we now require that it guarantees at least $m_{i}(p)$. We see from (4.3) that though such a strategy may not guarantee $v_{i}(p)$, $A_{i}$ will nevertheless get more by playing it than by passing (if this would be allowed).

That the limit of $v_{i}(n p) / n$ exists can be proved directly by an inequality given by Milnor [1, p. 294]:

$$
v_{i}(p)-v_{3-i}\left(p^{\prime}\right) \leqq v_{i}\left(p+p^{\prime}\right) \leqq v_{i}(p)+v_{\iota}\left(p^{\prime}\right) .
$$

We get

$$
v_{i}((m+n) p) \leqq v_{i}(m p)+v_{i}(n p),
$$

and the existence of the limit of $v_{i}(n p) / n$ follows (cf. [2], Erster Abschnitt, Aufgabe 98).

Another way of attacking our problem also leading to the number $m_{i}(p)$ will be used below. When a player shall move in a sum of games he chooses one game, say $G$, and there makes a move. Thereby he loses the possibility to make the move in one of the other games. If the value of this possibility is put equal to $t$ it is natural to compare the situation with the case when the player has to move in $G$ and pay the amount $t$ to the other player when moving. This will lead to the games $G_{t}$ and $G_{t}^{*}$ given in the next section. In this approach the value $m_{i}(p)$ is defined by induction on $l(p)$, thus by a finite procedure and not by a limit process.

Conventions for the figures. When giving examples of games by figures we use the following conventions. The positions are given by points and the moves indicated by segments joining them. A move by $A_{1}$ is a segment going down and to the left, a move by $A_{2}$ a segment going down and to the right. At an end position we put the value $k_{1}(p)$ and at any other position we put the two numbers $(m, \sigma)$, where $m=m_{1}(p)$ and $\sigma=\sigma(p)$ defined in the next section. Unless anything else is said, the game shall be played with the highest point as starting position.

EXAMPLE 1. Let $G$ be the game in Figure 1, and consider the sum of $n$ copies of $G$. First let us start at $p_{2}$ in all games. Then of course in about half of the games $A_{1}$ will get 7 and in the rest of them 3 . Hence the mean value $m_{1}\left(p_{2}\right)$ is $(7+3) / 2=5$. Analogously we get 
$m_{1}\left(p_{3}\right)=-1$. If all games are started from $p_{1}$, it can be proved that an optimal play by both players is to choose the moves from $p_{1}, p_{2}$, and

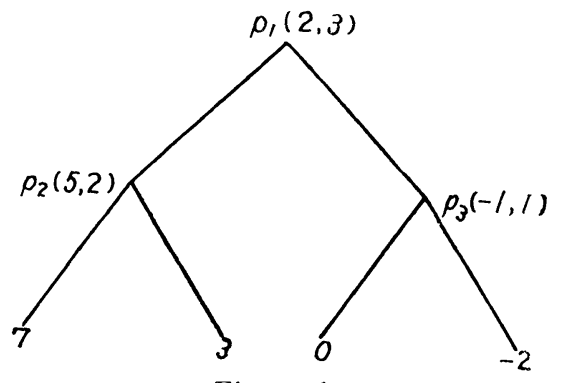

Figure 1 $p_{3}$ in this order of preference. Thus when both play optimally one move will first be made in all games. After these $n$ moves the players start attacking the positions $p_{2}$ in the games where $A_{1}$ made the move from $p_{1}$. At last the remaining games with positions $p_{3}$ are played. About $1 / 4$ of the games will end in each of the four end positions. Hence the mean value $m_{1}\left(p_{1}\right)$ is $(7+3+0-2) / 4=2$. The order of preference between $p_{1}, p_{2}, p_{3}$ is to be compared with the numbers $\sigma\left(p_{1}\right), \sigma\left(p_{2}\right), \sigma\left(p_{3}\right)$ which are defined in the next section. As given in the figure, $\sigma\left(p_{1}\right)=3$, $\sigma\left(p_{2}\right)=2, \sigma\left(p_{3}\right)=1$. The number $\sigma(p)$ is in a sense the value of the move from position $p$.

ExAMPLE 2. We change one of the payoff numbers in Figure 1 and get the game in Figure 2. Let us again consider the play of the sum of $n$ copies of the game. If all the games are started from $p_{1}$, the optimal play is now to choose the moves from $p_{1}, p_{2}, p_{3}$ in the order of preference: $p_{2}, p_{1}, p_{3}$, in accordance with the fact that $\sigma\left(p_{2}\right)=5, \sigma\left(p_{1}\right)=4$, and $\sigma\left(p_{3}\right)=1$. Thus if $A_{1}$ moves from $p_{1}$ to $p_{2}$ in a game, $A_{2}$ will immediately move in the same game. Thus all games with only one possible excep-

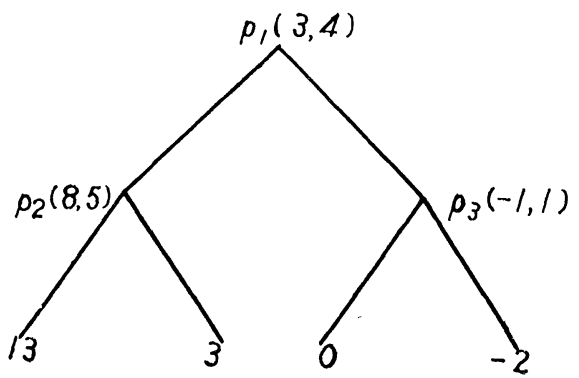

Figure 2 tion will end in the position with payoff $k_{1}(p)=3$. Thus $m_{1}\left(p_{1}\right)=3$. Only if $A_{2}$ has the first move one game will end in another end position, the one with $k_{1}(p)=0$.

5. The games $G_{t}$ and $G_{t}^{*}$. Let $G$ be a game satisfying as usual the condition (2.3). Let $t$ be a real number $\geqq 0$. When $l(p)=0$ put for $i=1,2$,

$$
\begin{aligned}
v_{i}(p ; t) & =k_{i}(p), \\
m_{i}(p) & =k_{i}(p), \\
\sigma(p) & =0 .
\end{aligned}
$$

For each $p$ with $l(p)>0$, we define four functions in $t: u_{1}(p ; t), u_{2}(p ; t)$, 
$v_{1}(p ; t), \quad v_{2}(p ; t)$ and three numbers $m_{1}(p), m_{2}(p)$, and $\sigma(p)$. They shall satisfy $(5.1)-(5.7)$.

(5.1) Each function $u_{i}(p ; t)$ and $v_{i}(p ; t)$ is a continuous function for $t \geqq 0$ with a derivative for all but a finite number of $t$-values. In each interval between these exception values the function is linear with derivative 0 or -1 . For $t$ greater than the exception values the function $u_{i}(p ; t)$ has derivative -1 and $v_{i}(p ; t)$ has derivative 0 ,

$$
\begin{gathered}
v_{i}(p ; 0)=v_{i}(p), \\
u_{i}(p ; t)=\max \left\{-v_{3-i}\left(p_{1} ; t\right) \mid p_{1} \in M_{i}(p)\right\}-t, \\
u_{i}(p ; 0)=v_{i}(p), \\
\sigma(p)=\min \left\{t \mid t \geqq 0, u_{1}(p ; t)+u_{2}(p ; t)=0\right\}, \\
m_{i}(p)=u_{i}(p ; \sigma(p)), \\
v_{i}(p ; t)=u_{i}(p ; t) \\
=m_{i}(p)
\end{gathered}
$$

We shall see below that these conditions are related to two games $G_{t}$ and $G_{t}^{*}$. Let us first show, however, that they define our functions and numbers by induction on $l(p)$.

For $l(p)=0$ the function $v_{i}(p ; t)$ is constant and equal to $v_{i}(p)$, hence it satisfies (5.1) and (5.2). Let $l(p)>0$ and suppose that for each $p_{1}$ with $l\left(p_{1}\right)<l(p)$ and in particular for each $p_{1} \in M_{i}(p)$ we have $v_{i}\left(p_{1} ; t\right)$ defined satisfying (5.1) and (5.2). Then $u_{i}(p ; t)$ can be defined by (5.3). By (5.1) for each $v_{3-i}\left(p_{1} ; t\right)$ we get immediately $(5.1)$ for $u_{i}(p ; t)$ and by (5.2) for each $v_{3-i}\left(p_{1} ; t\right)$ and by (2.2) we get (5.4). By (5.4) and (2.3) we have $u_{1}(p ; 0)+u_{2}(p ; 0) \geqq 0$ and by $(5.1)$ for $u_{i}(p ; t)$ we have $u_{1}(p ; t)+u_{2}(p ; t) \rightarrow-\infty$ when $t \rightarrow \infty$. Hence, since $u_{i}(p ; t)$ is continuous, the set in (5.5) is not vacuous and $\sigma(p)$ is defined and $\geqq 0$. Then (5.6) and (5.7) will define $m_{i}(p)$ and $v_{i}(p ; t)$. That $v_{i}(p ; t)$ satisfies (5.1) and (5.2) follows from the corresponding facts for $u_{i}(p ; t)$. Hence the induction will work.

EXAMPLe 3. We give in the diagram in Figure 4 the functions $u_{1}(p ; t), v_{1}(p ; t),-u_{2}(p ; t),-v_{2}(p ; t)$ for the game in Figure 3 and also the values $m_{1}(p)$ and $\sigma(p)$ for the same game.

Properties (5.1)-(5.7) give some further formulas. Since (5.1)-(5.7) are only known to be true for $l(p)>0$, we have to verify separately the case $l(p)=0$ each time we get a formula which has a meaning even in this case. Note that $u_{i}(p ; t)$ is not defined when $l(p)=0$. 


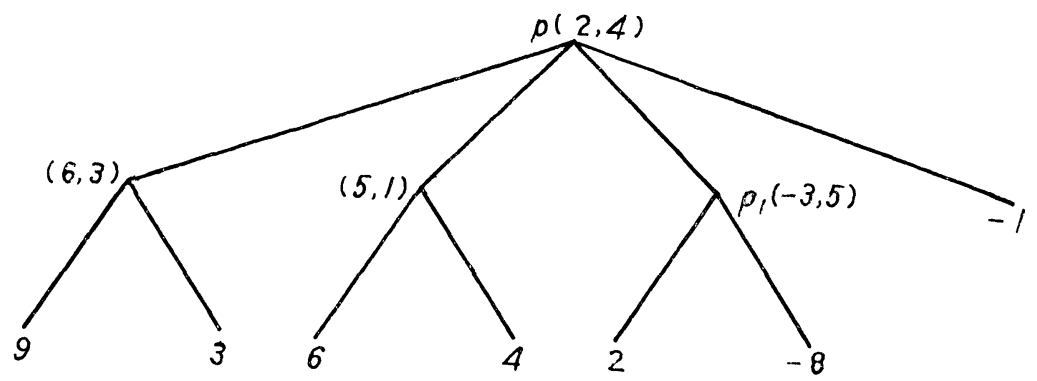

Figure 3

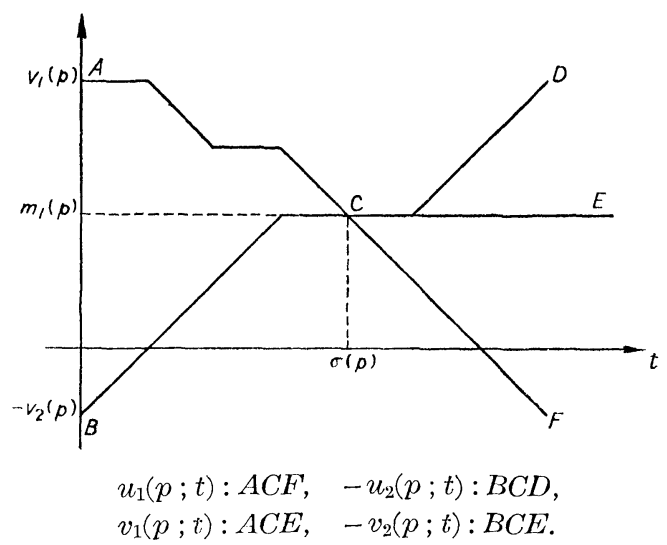

Figure 4

Since by $(5.1) u_{i}(p ; t)$ is a decreasing function, (5.6) and (5.7) give

$$
v_{i}(p ; t)=\max \left\{u_{i}(p ; t), m_{i}(p)\right\} .
$$

Hence, in particular

$$
v_{i}(p ; t) \geqq m_{i}(p) .
$$

By (5.5) and (5.6)

$$
m_{1}(p)+m_{2}(p)=0 .
$$

Both (5.9) and (5.10) are true also when $l(p)=0$ as is easily verified. For any $p$ they imply

$$
v_{1}(p ; t)+v_{2}(p ; t) \geqq 0 .
$$

By (5.2) and (5.9) we obtain

$$
v_{i}(p) \geqq m_{i}(p) .
$$

Since $v_{i}(p ; t)$ has derivative 0 or -1 , we have for $t_{1}<t_{2}$

$$
0 \leqq v_{i}\left(p ; t_{1}\right)-v_{i}\left(p ; t_{2}\right) \leqq t_{2}-t_{1} .
$$


Apply this for $t_{1}=0$ and $t_{2}=\sigma(p)$. Then by (5.2), (5.6), and (5.7)

$$
v_{i}(p) \leqq m_{i}(p)+\sigma(p) \text {. }
$$

Both (5.12) and (5.13) are also true when $l(p)=0$. For any $p$ they give a lower and an upper bound for $v_{i}(p)$.

We are now ready to define the two games $G_{t}$ and $G_{t}^{*}$ mentioned above. Both are defined for each $t \geqq 0$. They are played with the positions in $G$. The players play alternatively. But each time a player makes a move into a new position in $G$ he has to pay $t$ to the other player. Thus for large $t$ it will be expensive to make a move. Therefore we introduce a new possibility. When $A_{i}$ has the move in $G_{t}$ he is allowed to stop the game instead of moving. In $G_{t}^{*}$ the same possibility is open except in the starting position, where the player who begins really must move (and pay $t$ ). When $A_{i}$ stops at $p$ he collects $m_{i}(p)$. Then $A_{3-i}$ gets $m_{3-i}(p)$ by (5.10). The value of $G_{t}$ at $p$ is $v_{i}(p ; t)$ and the value of $G_{i}^{*}$ started at $p$ is $u_{i}(p ; t)$. This is seen by induction from (5.3) and (5.8).

For large $t$ it is a disadvantage to have to start in $G_{t}^{*}$. The starting player will make a move and pay $t$ and the other player will then immediately stop the game. Thus if $t$ is great enough the starting player will always lose. Thus $G_{t}^{*}$ does not satisfy (2.3). The game $G_{t}$, how ${ }^{-}$ ever, satisfies (2.3) as is seen from (5.11). In fact we have introduced the number $m_{i}(p)$ and the possibility to stop just in order to save this property. The number $m_{i}(p)$ is defined by (5.5) and (5.6) as the value of $G_{t}^{*}$ with starting position $p$, when $t$ has become so large that it is no more an advantage to have the first move in $G_{t}^{*}$. The lowest $t$-value of this kind is $\sigma(p)$.

6. The $t$-optimal moves. We will call a move in $G$ a $t$-optimal move if it is optimal in $G_{t}$. Thus $p_{\perp} \in M_{i}(p)$ is $t$-optimal if

$$
v_{i}(p ; t)=-v_{3-i}\left(p_{1} ; t\right)-t .
$$

There is a $t$-optimal move at $p$ for $A_{i}$ if $v_{i}(p ; t)=u_{i}(p ; t)$. Thus we get from (5.7) the following important fact: If $\sigma(p) \geqq t$ and if $p$ is not an end position there always exist $t$-optimal moves for both players.

If $\sigma(p) \leqq t$ we have $v_{i}(p ; t)=m_{i}(p)$, and an optimal play of $G_{t}$ is to stop the game at $p$ and collect $m_{i}(p)$.

Now study a sequence $p_{0}, p_{1}, p_{2}, \cdots, p_{l}$ of positions that develop when the players play alternatively and make $t$-optimal moves. If $\sigma\left(p_{l}\right)>t$, there are $t$-optimal moves at $p_{l}$. Therefore the sequence can be continued and we can go on in this way until we reach a position $p$ with $\sigma(p) \leqq t$. We suppose this already done, so that $\sigma\left(p_{l}\right) \leqq t$.

We want to get some formulas for $m_{i}\left(p_{k}\right), 0 \leqq k \leqq l$. Since all moves 
in the sequence are $t$-optimal we know that a player cannot get more when playing $G_{t}$ by stopping at a position $p_{k}, k<l$, than by moving into $p_{k+1}$. Thus if $A_{i}$ makes the first move and if we put $v_{i}\left(p_{J} ; t\right)=v$, we get

$$
\begin{array}{cl}
m_{i}\left(p_{2 k}\right) \leqq v & \text { if } 0 \leqq 2 k<l, \\
m_{i}\left(p_{2 k+1}\right) \geqq v+t & \text { if } 1 \leqq 2 k+1<l,
\end{array}
$$

where the term $+t$ in (6.3) is the amount $A_{i}$ shall have when the game is stopped after an odd number of moves as a compensation for the fact that he has made one more move than $A_{3-i}$, each player paying $t$ when moving in $G_{t}$. Since $\sigma\left(p_{l}\right) \leqq t$, an optimal play at $p_{l}$ in $G_{t}$ is to stop the game. Hence

$$
\begin{array}{ll}
m_{i}\left(p_{l}\right)=v & \text { if } l \text { is even, } \\
m_{i}\left(p_{l}\right)=v+t & \text { if } l \text { is odd. }
\end{array}
$$

Formulas (6.2)-(6.5) could also have been deduced from (6.1). Since all moves are $t$-optimal we get $v_{i}\left(p_{0} ; t\right)=-v_{3-i}\left(p_{1} ; t\right)-t=v_{i}\left(p_{2} ; t\right)=$ $-v_{3-i}\left(p_{3} ; t\right)-t=\cdots$ and $(6.2)-(6.5)$ follow if we apply (5.9) and (5.10) and the fact that since $\sigma\left(p_{l}\right) \leqq t$, we have by $(5.7), m_{j}\left(p_{l}\right)=v_{j}\left(p_{l} ; t\right)$ for $j=1,2$.

EXAMPLE 4. The game in Figure 5 shows that strong inequality may

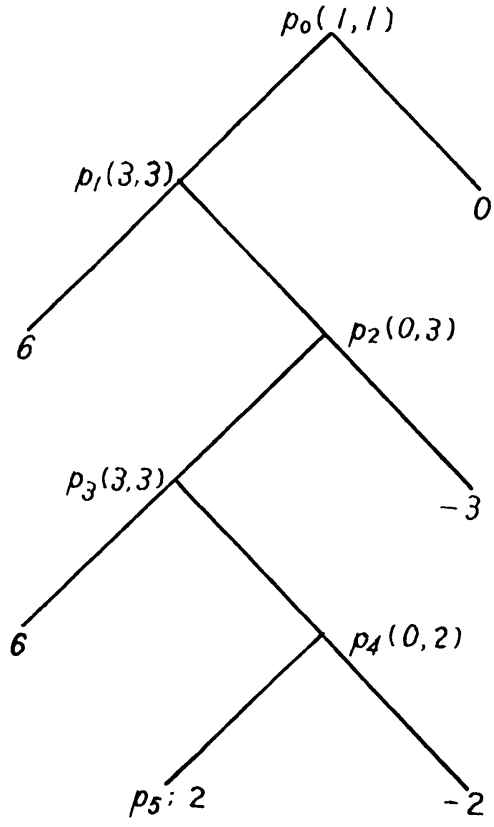

Figure 5 hold in (6.2) and (6.3). All the moves which lead from $p_{0}$ to $p_{5}$ are 1-optimal and $v=v\left(p_{0} ; 1\right)=1$.

Let now only one player make $t$ optimal moves when playing $G_{t}$. He will get at least as much as when also the other player makes $t$-optimal moves. Thus we can get some formulas corresponding to $(6.2)-(6.5)$. We put them together into two lemmas.

Lemma 1. Let $p_{0}, p_{1}, \cdots, p_{l}$ be a sequence of positions in $G$ such that $p_{2 k+1} \in$ $M_{i}\left(p_{2 k}\right)$, where $p_{2 k+1}$ is a t-optimal move at $p_{2 k}$, and such that $p_{2 k+2} \in M_{3-i}\left(p_{2 k+1}\right)$. Then if $v_{i}\left(p_{0} ; t\right)=v$, we have

$$
\begin{aligned}
& m_{i}\left(p_{2 k+1}\right) \geqq v+t, \\
& \quad m_{i}\left(p_{l}\right) \geqq v \\
& \quad \text { if } \sigma\left(p_{l}\right) \leqq t \text { and } l \text { is even. }
\end{aligned}
$$


Lemma 2. Let $p_{0}, p_{1}, \cdots, p_{l}$ be a sequence of positions in $G$ such that $p_{2 k+1} \in M_{i}\left(p_{2 k}\right)$ and $p_{2 k+2} \in M_{3-i}\left(p_{2 k+1}\right)$, where $p_{2 k+2}$ is a t-optimal move at $p_{2 k+1}$. Then if $v_{i}\left(p_{0} ; t\right)=v$ we have

$$
\begin{aligned}
& m_{i}\left(p_{2 k}\right) \leqq v, \\
& m_{i}\left(p_{l}\right) \leqq v+t \quad \text { if } \sigma\left(p_{l}\right) \leqq t \text { and } l \text { is odd. }
\end{aligned}
$$

7. The mean strategies for sum games. We now go to our main subject, sums of games.

TheOREM 1. Let us start the games $G_{1}, \cdots, G_{n}$ in positions $q_{1}, \cdots, q_{n}$. Put

$$
\begin{aligned}
m_{i} & =m_{i}\left(q_{1}\right)+\cdots+m_{i}\left(q_{n}\right), \\
\sigma & =\max \left\{\sigma\left(q_{r}\right) \mid 1 \leqq r \leqq n\right\} .
\end{aligned}
$$

Then the value $v_{i}\left(q_{1}+\cdots+q_{n}\right)$ for $A_{i}$ when he starts at $q_{1}+\cdots+q_{n}$ in $G_{1}+\cdots+G_{n}$ satisfies

$$
m_{i} \leqq v_{i}\left(q_{1}+\cdots+q_{n}\right) \leqq m_{i}+\sigma .
$$

Proof. We proceed by induction on $l\left(q_{1}+\cdots+q_{n}\right)$. When $l\left(q_{1}+\cdots+q_{n}\right)=0$, all $q_{r}$ are end positions and our theorem follows directly from $m_{i}\left(q_{r}\right)=k_{i}\left(q_{r}\right)$ and $\sigma\left(q_{r}\right)=0$. By (2.1) we know that if one or several moves are made from $q_{1}+\cdots+q_{n}$ we come to a position, say $p_{1}+\cdots+p_{n}$, with

$$
l\left(p_{1}+\cdots+p_{n}\right)<l\left(q_{1}+\cdots+q_{n}\right) .
$$

Hence when proving our theorem we may assume that it is true for all positions obtainable from $q_{1}+\cdots+q_{n}$ by one or several moves.

By symmetry we may specialize in the proof so that $i=1$, i.e. $A_{1}$ makes the first move. We then want a strategy for him that secures the amount $m_{1}$ and a strategy for $A_{2}$ such that $A_{1}$ cannot get more than $m_{1}+\sigma$. These strategies can be formulated together.

$(\alpha)$ Always make a $\sigma$-optimal move in one of the games $G_{1}, \cdots, G_{n}$.

( $\beta$ ) Except for the first move, play in the game, in which the other player has just played.

In general it will not be possible to follow this strategy through the whole play of the game, since there are not $\sigma$-optimal moves in all positions. The strategy shall therefore be used during a period in the beginning of the play. In the position at the end of this period the induction hypothesis will be used. 'The length of the period depends upon the moves made. We give two possibilities to end the period. 
$\left(\gamma_{1}\right)$ The other player plays in a game $G_{r}$ and there leaves a position $p_{r}$ with $\sigma\left(p_{r}\right) \leqq \sigma$.

$\left(\gamma_{2}\right)$ Positions $p_{r}, 1 \leqq r \leqq n$, are reached for which $\sigma\left(p_{r}\right) \leqq \sigma$.

We have to show that a player can follow $(\alpha)$ and $(\beta)$ until $\left(\gamma_{1}\right)$ or $\left(\gamma_{2}\right)$ occurs. We first see that $A_{1}$ always can make his first move. In fact, by the definition of $\sigma$ there is a $q_{r}$. with $\sigma\left(q_{r}\right)=\sigma$. Thus there is a $\sigma$-optimal move in $G_{r}$. For all later moves the player following the strategy shall play in the position $p_{r}$. which the other player has just left. Then if $\left(\gamma_{1}\right)$ does not occur, $\sigma\left(p_{r}\right)>\sigma$ and there is a $\sigma$-optimal move at $p_{r}$. Hence the game can be continued until $\left(\gamma_{1}\right)$ occurs or until the player following the strategy ends the whole sum game by playing into an end position. Then $\sigma\left(p_{r}\right)=0$ for all games $G_{r}$ and $\left(\gamma_{2}\right)$ is satisfied. Hence it is possible to follow $(\alpha)$ and $(\beta)$ until $\left(\gamma_{1}\right)$ or $\left(\gamma_{2}\right)$ occurs.

In order to be able to use the induction hypothesis we have to compare $m_{1}$ with

$$
m_{1}\left(p_{1}\right)+\cdots+m_{1}\left(p_{n}\right),
$$

where $p_{r}$ is the position in $G_{r}$ at the end of the period. Therefore we first compare $m_{1}\left(q_{r}\right)$ with $m_{1}\left(p_{r}\right)$ for each $r$. Hence we are interested in those moves in the period that are made in $G_{r}$. Note that when at least one player follows the strategy, $(\beta)$ implies that these moves are played alternatively by the players. Thus for each $G_{r}$ we are able to apply Lemmas 1 and 2 of the preceding section with $t=\sigma$. Since $\sigma \geqq \sigma\left(q_{r}\right)$, the number $v=v_{i}\left(q_{r} ; \sigma\right)$ in these lemmas is $=m_{i}\left(q_{r}\right)$.

Let first $A_{1}$ follow the strategy. Denote by $p_{r}$ the position in $G_{r}$ at the end of the period. Then if the move into $p_{r}$ is made by $A_{2}$, we know, since $A_{1}$ follows $(\beta)$, that this move is the last move in the period, and whether the period ends with $\left(\gamma_{1}\right)$ or $\left(\gamma_{2}\right)$ we get $\sigma\left(p_{r}\right) \leqq \sigma$ in this game $G_{r}$. Using the fact (5.10) for $q_{r}$ and $p_{r}, 1 \leqq r \leqq n$, we apply Lemma 1 with $i=1$ and Lemma 2 with $i=2$. Then (6.6), (6.7), (6.8), and (6.9) imply respectively the following four formulas, depending upon who makes the first move and the last move in $G_{r}$.

$$
\begin{array}{ll}
m_{1}\left(p_{r}\right) \geqq m_{1}\left(q_{r}\right)+\sigma & A_{1} \text { first and last move, } \\
m_{1}\left(p_{r}\right) \geqq m_{1}\left(q_{r}\right) & A_{1} \text { first move, } A_{2} \text { last move, } \\
m_{1}\left(p_{r}\right) \geqq m_{1}\left(q_{r}\right) & A_{2} \text { first move, } A_{1} \text { last move, } \\
m_{1}\left(p_{r}\right) \geqq m_{1}\left(q_{r}\right)-\sigma & A_{2} \text { first and last move. }
\end{array}
$$

We add the trivial fact

$$
m_{1}\left(p_{r}\right)=m_{1}\left(q_{r}\right) \quad \text { if no move is made in } G_{r} \text {. }
$$

Formulas (7.1)-(7.5) can be taken together in one formula 


$$
m_{1}\left(p_{r}\right) \geqq m_{1}\left(q_{r}\right)+l_{1 r} \sigma-l_{2 r} \sigma,
$$

where $l_{i r}$ is the number of moves made by $A_{i}$ in $G_{r}$ during the period. Let us take the sum of the inequalities (7.6) for all $r$. Then

$$
m_{1}\left(p_{1}\right)+\cdots+m_{1}\left(p_{n}\right) \geqq m_{1}+l_{1} \sigma-l_{2} \sigma,
$$

where $l_{i}$ is the number of moves made by $A_{i}$ during the period.

If the number of moves in the period is even we have $l_{1}=l_{2}$. $A_{1}$ who makes the first move in the period shall also make the first move after the period (if there is any move to be made). $A_{1}$ can play so after the period that he secures $v_{1}\left(p_{1}+\cdots+p_{n}\right)$. By the induction hypothesis this is $\geqq m_{1}\left(p_{1}\right)+\cdots+m_{1}\left(p_{n}\right)$ which by $(7.7)$ is $\geqq m_{1}$. Hence we have shown that $A_{1}$ has been able to play from $q_{1}+\cdots+q_{n}$ so as to secure $m_{1}$, and the left-hand inequality of our theorem is proved in this case.

We also have to consider the case that the period contains an odd number of moves. Then since $A_{1}$ makes the first move he also makes the last move and the period is not ended by $\left(\gamma_{1}\right)$, hence by $\left(\gamma_{2}\right)$. Thus $\sigma\left(p_{r}\right) \leqq \sigma$ for each $G_{r}$. We have now $l_{1}=l_{2}+1$. $A_{1}$ can play so after the period that he secures $-v_{2}\left(p_{1}+\cdots+\mathrm{By} p_{n}\right)$. the induction hypothesis, by $\sigma\left(p_{r}\right) \leqq \sigma$, and by (7.7) we get

$$
\begin{aligned}
-v_{2}\left(p_{1}+\cdots+p_{n}\right) & \geqq-m_{2}\left(p_{1}\right)-\cdots-m_{2}\left(p_{n}\right)-\max \left\{\sigma\left(p_{r}\right)\right\} \\
& \geqq-m_{2}\left(p_{1}\right)-\cdots-m_{2}\left(p_{n}\right)-\sigma \\
& =m_{1}\left(p_{1}\right)+\cdots+m_{1}\left(p_{n}\right)-\sigma \\
& \geqq m_{1} .
\end{aligned}
$$

Hence the left-hand inequality of the theorem is proved even in this case.

In order to prove the right-hand inequality of the theorem we let $A_{2}$ follow the strategy. Then by $(\beta) A_{1}$ makes the first move in each $G_{r}$ (if there is any move in $G_{r}$ during the period). Lemma 2 with $i=1$ gives now depending upon who makes the last move in $G_{r}$.

$$
\begin{array}{ll}
m_{1}\left(p_{r}\right) \leqq m_{1}\left(q_{r}\right) & A_{2} \text { last move }, \\
m_{1}\left(p_{r}\right) \leqq m_{1}\left(q_{r}\right)+\sigma & A_{1} \text { last move. }
\end{array}
$$

Proceeding as above we get a formula like (7.7), namely

$$
m_{1}\left(p_{1}\right)+\cdots+m_{1}\left(p_{n}\right) \leqq m_{1}+l_{1} \sigma-l_{2} \sigma \text {. }
$$

If the period contains an odd number of moves, $l_{1}=l_{2}+1$. $A_{2}$ makes then the first move after the period (if there is any move to be made). He can therefore play so that $A_{1}$ gets at most $-v_{2}\left(p_{1}+\cdots+p_{n}\right)$. By the induction hypothesis and by (7.10) 


$$
\begin{aligned}
-v_{2}\left(p_{1}+\cdots+p_{n}\right) & \leqq-m_{2}\left(p_{1}\right)-\cdots-m_{2}\left(p_{n}\right) \\
& =m_{1}\left(p_{1}\right)+\cdots+m_{1}\left(p_{n}\right) \\
& \leqq m_{1}+\sigma,
\end{aligned}
$$

so that the right-hand inequality is proved in this case.

Finally if the period contains an even number of moves, $l_{1}=l_{2}$, and the period ends by $\left(\gamma_{2}\right)$, so that $\sigma\left(p_{r}\right) \leqq \sigma$. Then $A_{1}$ gets at most $v_{1}\left(p_{1}+\cdots+p_{n}\right)$ and by the induction hypothesis and by (7.10)

$$
\begin{aligned}
v_{1}\left(p_{1}+\cdots+p_{n}\right) & \leqq m_{1}\left(p_{1}\right)+\cdots+m_{1}\left(p_{n}\right)+\max \left\{\sigma\left(p_{r}\right)\right\} \\
& \leqq m_{1}\left(p_{1}\right)+\cdots+m_{1}\left(p_{n}\right)+\sigma \\
& \leqq m_{1}+\sigma
\end{aligned}
$$

and the right-hand inequality is proved even in this case.

This completes the proof of Theorem 1.

In the proof just completed the strategy given by $(\alpha)$ and $(\beta)$ is used only in a period in the beginning of the play. When this period is ended we have used the induction hypothesis in the proof of the theorem. This means, however, that we shall start counting a new period and then again apply $(\alpha)$ and $(\beta)$. Continuing in this way we get the following consequence of the proof of Theorem 1 .

THeORem 2. Make the same assumptions as in Theorem 1. Suppose one player, $A_{k}$, follows a strategy satisfying (a)-(d) below. Then $A_{i}$, the player making the first move. will get at least $m_{i}$ when $k=i$ and at most $m_{i}+\sigma$ when $k=3-i$.

(a) Divide the moves made by the two players into periods.

(b) For each period let $\tau$ be the maximum of $\sigma\left(p_{r}\right)$ for the positions $p_{r}$ at the beginning of the period. With this $\tau$ defined for a period, always make r-optimal moves in the period.

(c) Except for the first move in a period play in the game in which the other player has just played.

(d) Start counting a new period when one of the following two situations occurs,

$\left(\mathrm{d}_{1}\right)$ the other player plays in $G_{r}$ into a position $p_{r}$ with $\sigma\left(p_{r}\right) \leqq \tau$,

$\left(d_{2}\right)$ positions $p_{r}$ with $\sigma\left(p_{r}\right) \leqq \tau$ are reached in all $G_{r}, 1 \leqq r \leqq n$.

We call the strategies that satisfies (a)-(d) of this theorem mean strategies.

8. Properties of $m_{i}(p)$ and $\sigma(p)$. By Theorem 1 we easily prove the fact that the sum of games satisfying (2.3) also satisfies (2.3) (proved by Milnor [1, p. 294]). In fact by Theorem 1

$$
v_{i}\left(q_{1}+\cdots+q_{n}\right) \geqq m_{i} .
$$


Since $m_{1}\left(q_{r}\right)+m_{2}\left(q_{r}\right)=0$ for each $r$, we have $m_{1}+m_{2}=0$. Hence

$$
v_{1}\left(q_{1}+\cdots+q_{n}\right)+v_{2}\left(q_{1}+\cdots+q_{n}\right) \geqq 0,
$$

which is (2.3) for $G_{1}+\cdots+G_{n}$.

Thus $G_{1}+\cdots+G_{n}$ is a game of the kind described in $\S 2$. We can therefore apply $\S 5$ and define e.g. $u_{i}\left(q_{1}+\cdots+q_{n} ; t\right), m_{1}\left(q_{1}+\cdots+q_{n}\right)$, and $\sigma\left(q_{1}+\cdots+q_{n}\right)$.

Theorem 3. Let us start the games $G_{1}, \cdots, G_{n}$ in positions $q_{1}, \cdots, q_{n}$. Then

$$
\begin{aligned}
m_{i}\left(q_{1}+\cdots+q_{n}\right) & =m_{i}\left(q_{1}\right)+\cdots+m_{i}\left(q_{n}\right), \\
\sigma\left(q_{1}+\cdots+q_{n}\right) & \leqq \max \left\{\sigma\left(q_{r}\right) \mid 1 \leqq r \leqq n\right\} .
\end{aligned}
$$

The right-hand side of these formulas is just $m_{i}$ and $\sigma$ respectively defined in Theorem 1.

Proof. We need the following lemma.

LEMMA 3.

$$
u_{i}\left(q_{1}+\cdots+q_{n} ; \sigma\right)=m_{i} \text { when } l\left(q_{1}+\cdots+q_{n}\right)>0 .
$$

Before proving the lemma let us see that Theorem 3 follows from it. If $l\left(q_{1}+\cdots+q_{n}\right)=0,(8.1)$ and (8.2) are certainly true. If $l\left(q_{1}+\cdots+q_{n}\right)>0$ we get from Lemma 3 , since $m_{1}+m_{2}=0$,

$$
u_{1}\left(q_{1}+\cdots+q_{n} ; \sigma\right)+u_{2}\left(q_{1}+\cdots+q_{n} ; \sigma\right)=0 .
$$

Then (8.2) follows from (5.5). We also see from (5.5) and the fact that $u_{i}\left(q_{1}+\cdots+q_{n} ; t\right), i=1,2$, are decreasing functions in $t$, that they are constant in the interval $\left(\sigma\left(q_{1}+\cdots+q_{n}\right), \sigma\right)$. Then (8.1) follows from (5.6) and Lemma 3.

Proof of Lemma 3. The proof will be somewhat similar to that of Theorem 1. Without losing generality we put $i=1$. We make the induction hypothesis that Theorem 3 is true for all $p_{1}+\cdots+p_{n}$ obtainable from $q_{1}+\cdots+q_{n}$ by one or several moves. We will prove

$$
\begin{aligned}
& u_{1}\left(q_{1}+\cdots+q_{n} ; \sigma\right) \geqq m_{1}, \\
& u_{1}\left(q_{1}+\cdots+q_{n} ; \sigma\right) \leqq m_{1} .
\end{aligned}
$$

Of course they together will give Lemma 3 . The number $u_{1}\left(q_{1}+\cdots+q_{n} ; \sigma\right)$ is the value for $A_{1}$ in the game $\left(G_{1}+\cdots+G_{n}\right)_{\sigma}^{*}$. To prove (8.3) and (8.4) we define strategies for $A_{1}$ and $A_{2}$ in this game: Follow $(\alpha)$ and $(\beta)$ of the proof of Theorem 1. Unless the other player stops the game in 
some position, continue until $\left(\gamma_{1}\right)$ occurs and then stop the game. When the game is stopped at $p_{1}+\cdots+p_{n}, A_{1}$ collects $m_{1}\left(p_{1}+\cdots+p_{n}\right)$. If then $A_{1}$ has made $l_{1}$ and $A_{2} l_{2}$ moves $\left(l_{1}=l_{2}\right.$ or $\left.l_{1}=l_{2}+1\right), A_{1}$ has paid $l_{1} \sigma$ to $A_{2}$ and got $l_{2} \sigma$ from him. Hence the result will be that $A_{1}$ gets

$$
m_{1}\left(p_{1}+\cdots+p_{n}\right)-l_{1} \sigma+l_{2} \sigma .
$$

Since by the induction we may apply Theorem 3 , this is equal to

$$
m_{1}\left(p_{1}\right)+\cdots+m_{n}\left(p_{n}\right)-l_{1} \sigma+l_{2} \sigma .
$$

Thus in order to prove (8.3) and (8.4) we only need to verify that (7.7) and (7.10) are true when $A_{1}$ and $A_{2}$ respectively use the strategy described above.

Let $A_{1}$ follow the strategy, and let $p_{r}$ be the position in $G_{r}$. when the game is stopped. Then if the move into $p_{r}$ is made by $A_{2}$, we know since $A_{1}$ follows $(\beta)$, that this is the last move made before the game is stopped by $A_{1}$. Hence $\left(\gamma_{1}\right)$ is true, and we have $\sigma\left(p_{r}\right) \leqq \sigma$ for this game $G_{r}$. The proof of the formulas (7.1)-(7.4) now follows as in the proof of Theorem 1, and (7.7) will again be a consequence of these formulas. Hence we have given a strategy for $A_{1}$ in $\left(G_{1}+\cdots+G_{n}\right)_{\sigma}^{* k}$ which secures $m_{1}$. Thus (8.3) is proved.

Similarly if $A_{2}$ follows the strategy, we verify (7.8) and (7.9) thereby proving (7.10). Thus we have given a strategy for $A_{2}$ in $\left(G_{1}+\cdots+G_{n}\right)_{\sigma}^{*}$ such that $A_{1}$ gets $\leqq m_{1}$. This proves (8.4). Thus Lemma 3 is proved and also Theorem 3 .

Theorem 3 can be looked upon as a sharper form of Theorem 1 . In fact we get Theorem 1 from Theorem 3 simply by applying (5.12) and (5.13) for $p=q_{1}+\cdots+q_{n}$.

Let now the games $G_{1}, \cdots, G_{n}$ be $n$ copies of one and the same game $G$ and let $p_{1}, \cdots, p_{n}$ correspond to $p$ in $G$. We write $n p$ for $p_{1}+\cdots+p_{n}$. By Theorem 1

$$
n m_{i}(p) \leqq v_{i}(n p) \leqq n m_{i}(p)+\sigma(p) .
$$

Divide by $n$ and let $n \rightarrow \infty$. Then, because of (5.10), we get the following result.

THEOREM 4. The two expressions

$$
\frac{1}{n} v_{i}(n p) \quad \text { and } \quad \frac{1}{n}\left(-v_{3-i}(n p)\right)
$$

which represent the msan value for $A_{i}$ in the sum of $n$ equal games when he or the other player has the first move, both tend to the same limit $m_{i}(p)$ when $n \rightarrow \infty$.

This theorem justifies the name mean value for the number $m_{i}(p)$. 
The name mean strategies for the strategies described in Theorem 2 is chosen, since it secures the mean value for the player who makes the first move.

We know by Theorem 3 that

$$
m_{i}\left(p_{1}+\cdots+p_{n}\right)=m_{i}\left(p_{1}\right)+\cdots+m_{i}\left(p_{n}\right)
$$

and get from (5.10) and (5.12)

$$
-v_{3-i}(p) \leqq m_{i}(p) \leqq v_{i}(p) \text {. }
$$

Let us show that the two properties (8.5) and (8.6) determine $m_{i}(p)$ uniquely. Let $m(p)$ be given for all $p$ satisfying (8.5) and (8.6). We get

$$
-v_{3-i}(n p) \leqq n m(p) \leqq v_{i}(n p) \text {. }
$$

Divide by $n$ and let $n \rightarrow \infty$. Then, by Theorem 4 we get $m(p)=m_{i}(p)$, showing the uniqueness of $m_{i}(p)$.

\section{Both players use mean strategies.}

THEOREM 5. Let in a sum $G_{1}+\cdots+G_{n}$ both players follow a mean strategy, such as described by (a)-(d) in Theorem 2. Then

(1) the players will count the same periods,

(2) in each period both players will make all their moves in only one of the games $G_{r}$,

(3) the number $\tau$ defined by (b) of Theorem 2 is a decreasing function of the period,

(4) if to $m_{i}\left(q_{1}\right)+\cdots+m_{i}\left(q_{n}\right)$, where $q_{r}$ is the starting position of the game $G_{r}, 1 \leqq r \leqq n$, we add $\tau$ for each move $A_{i}$ makes and $-\tau$ for each move $A_{3-i}$ makes, where $\tau$ is defined by (b) for the period containing the move, then the result will be $A_{i}$ 's payoff.

Proof. Here (1) will follow by induction if we show that the first period ends at the same moment for both players. When both players play in their first periods (c) implies that they both move in the same game, say in $G_{s}$. Then for $r \neq s, p_{r}=q_{r}$ for all positions $p_{1}+\cdots+p_{n}$ that are reached in the period and therefore since $\sigma\left(q_{r}\right) \leqq \sigma$ by the definition of $\sigma$ (see Theorem 1 ), we get $\sigma\left(p_{r}\right) \leqq \sigma, r \neq s$. Thus when $\left(\mathrm{d}_{1}\right)$ occurs for one player $\left(d_{2}\right)$ also occurs and since $\left(d_{2}\right)$ is symmetric with respect to the two players the first period will be the same for both players. This proves (1).

When we know that the players count the same periods, (2) is a simple consequence of (c). (3) follows from the fact that each period ends with $\left(d_{2}\right)$.

To prove (4) it will be sufficient to show that if in $G_{r}, q_{r}$ is the 
position at the beginning of a period and $p_{r}$ is the position at the end of the same period then whether $A_{i}$ or $A_{3-i}$ starts the period,

$$
m_{i}\left(p_{1}\right)+\cdots+m_{i}\left(p_{n}\right)=m_{i}\left(q_{1}\right)+\cdots+m_{i}\left(q_{n}\right)+l_{i} \tau-l_{3-i} \tau,
$$

where $l_{i}$ is the number of moves by $A_{i}$ in the period. Since $p_{r}=q_{r}$ for $r \neq s$, where $G_{s}$ is the game in which all moves are made during the period, (9.1) reduces to

$$
m_{i}\left(p_{s}\right)=m_{i}\left(q_{s}\right)+l_{i} \tau-l_{3-i} \tau .
$$

If $A_{i}$ makes the first move in the period, (9.2) follows from (6.4) and (6.5). In fact these two formulas are proved for the case when both players make $t$-optimal moves until a position $p_{l}$ is reached with $\sigma\left(p_{l}\right) \leqq t$. But putting $t=\tau$ we get in our case by $\left(d_{2}\right)$ that for the final position $p_{s}$ of the period, $\sigma\left(p_{s}\right) \leqq \tau$.

If $A_{3-i}$ makes the first move in the period, (9.2) is just proved with $3-i$ substituted for $i$. However, the formula thus obtained reduces to (9.2) by the use of $m_{i}(p)+m_{3-i}(p)=0$.

Thus Theorem 5 is proved.

Since $\tau$ is decreasing we see by (4) of Theorem 5 that $A_{i}$ 's payoff is the sum of $m_{i}=m_{i}\left(q_{1}\right)+\cdots+m_{i}\left(q_{n}\right)$ and a sequence of terms with alternating signs and decreasing modules. If $A_{i}$ starts playing, the first term is positive and equal to $\sigma=\max \left\{\sigma\left(q_{r}\right)\right\}$ and the sum of the terms in the sequence is therefore $\geqq 0$ and $\leqq \sigma$, and $A_{i}$ will get at least $m_{i}$ and at most $m_{i}+\sigma$. This last result is of course contained in Theorem 2. Theorem 2 says even more, since it says that a mean strategy always guarantees a certain amount even if used against a player which plays any strategy, e.g. an optimal strategy.

10. Some examples. Conditions (a)-(d) of Theorem 2 do not in general determine a unique strategy. There are still some choices which the player may use to get as good result as possible. Thus there may be different $\tau$-optimal moves in the same game and, when the first move of a period shall be made, there may be several games in which there are $\tau$-optimal moves. In this connection it may be worth while to notice that there may be a $\tau$-optimal move even in a position $p$ with $\sigma(p)<\tau$. The number $\tau$ is determined as the maximum of $\sigma\left(p_{r}\right), 1 \leqq r \leqq n$ when the period starts, but it is not necessary to start the period in one of the games for which $\sigma\left(p_{r}\right)$ reaches this maximum. There may be $\tau$-optimal moves even in other games.

ExAmple 5. Let us study the game given in Figure 3. The move $p_{1} \in M_{2}(p)$ is $t$-optimal for $A_{2}$ even when $4<t \leqq 5$. In fact for these $t$-values $u_{2}(p ; t)=v_{2}(p ; t)=m_{2}(p)$ so that there must be a $t$-optimal move for $A_{2}$. 
If a position has to be played in optimal way it is unimportant if this position is the starting position of the game or if it is a position which has developed during the play. This is not the case when mean strategies are used.

Example 6 . Compare the game in Figure 6 started by $A_{2}$ and the game in Figure 7 started by $A_{1}$. When $A_{2}$ has moved into $p_{1}$ in Figure 6 the situation for $A_{1}$ will be the same as when he starts in $p_{1}$ in Figure

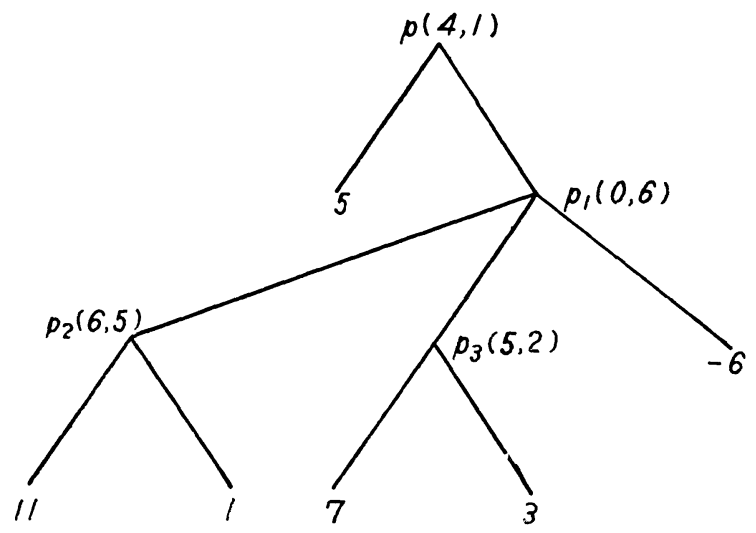

Figure 6

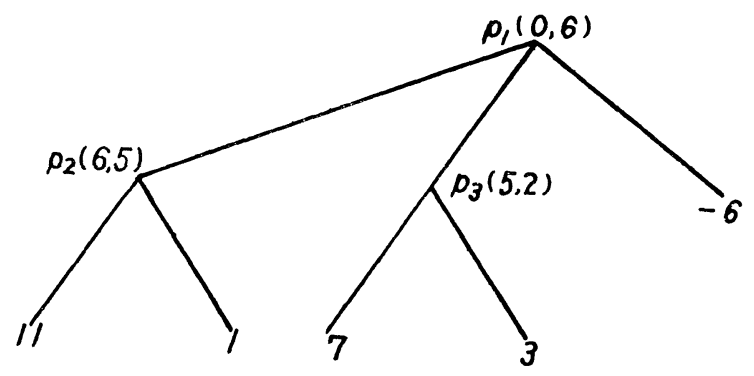

Figure 7

7. However, playing a mean strategy he will handle the two cases in different way. In Figure $6 A_{1}$ plays in a period with $\tau=1$. $\mathrm{He}$ will therefore make a 1-optimal move, the one into $p_{3}$. In Figure 7 he just starts a period with $\tau=6$ and moves into $p_{2}$.

This difference may be explained thus. The move recommended by a mean strategy shall be a good move when played in the sum of $n$ copies of the game. We see readily that in $n$ copies of the game in Figure 6 the move into $p_{3}$ is the correct answer to $A_{2}$ 's move into $p_{1}$. If $A_{1}$ always moves into $p_{2}$ he gets only about $5 \frac{n}{2}+1 \frac{n}{2}=3 n$ though $m_{1}(p)=4$. In $n$ copies of the game in Figure 7 the move into $p_{2}$ is 
correct. If $A_{1}$ always moves into $p_{3}$ he gets about $(-6) \frac{n}{2}+7 \frac{n}{4}+3 \frac{n}{4}=$ $-\frac{n}{2}$ though $m_{1}\left(p_{1}\right)=0$.

In a sense (4) of Theorem 5 means that the value of making a move is equal to the number $\tau$ for the period containing the move, where $\tau$ is $\max \left\{\sigma\left(p_{r}\right)\right\}$ at the beginning of the period. One may try to change the rules for a mean strategy by requiring each move to be played at the position $p$ where $\sigma(p)$ is highest. The following example shows, however, that such a play does not guarantee the mean value.

EXAMPle 7. Consider the sum game given in Figure 8. Suppose that $A_{1}$ starts and plays in the left game and that $A_{2}$ answers in the right game. Then $\sigma(p)=7$ in the left game and $\sigma(p)=6$ in the right
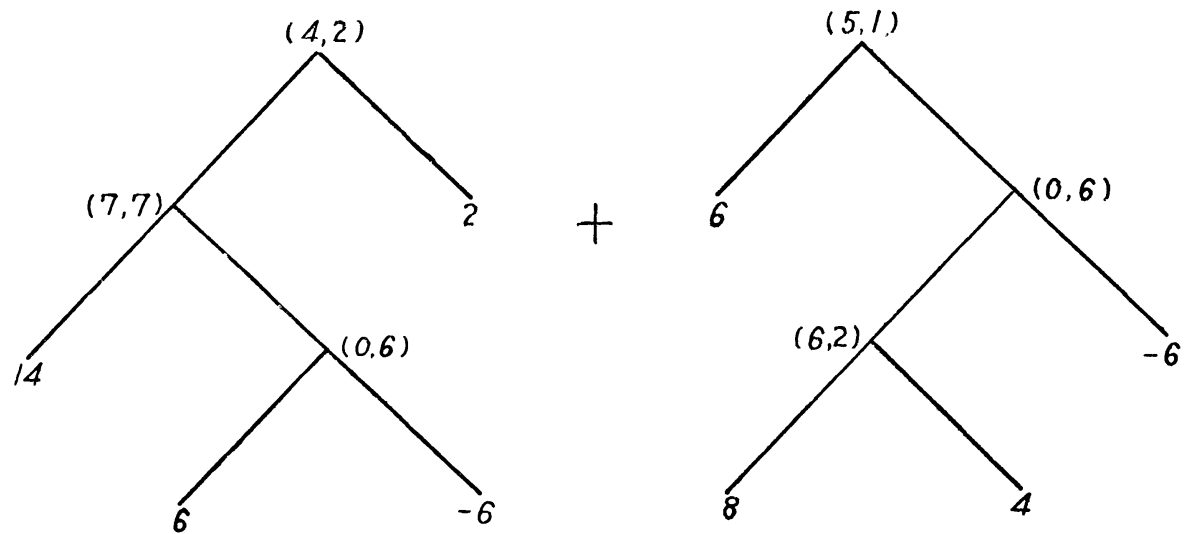

Figure 8

game. But if $A_{1}$ plays in the left game, where $\sigma(p)$ is highest he will get only $14+(-6)=8$ which is less than the mean value $4+5=9$. In fact $A_{1}$ 's second move is made in a period with $\tau=2$. Hence if he follows (a)-(d) he shall play a 2-optimal move in the game where the other player has just played, i.e. he shall play in the right game. Then he will get at least $6+4=10$ which is more than the mean value 9 .

Let us in a final example show that an optimal move in a sum game need not be $t$-optimal for any $t$ in the summand $G$ in which it is made. Hence this move can never be recommended by a mean strategy.

ExAmple 8. The optimal move for $A_{1}$ in the sum game in Figure 9 is the move into $p_{1}$ in the left game, the mean strategy move is the move in the right game. The move into $p_{1}$ is never $t$-optimal in the left game for any $t$. 

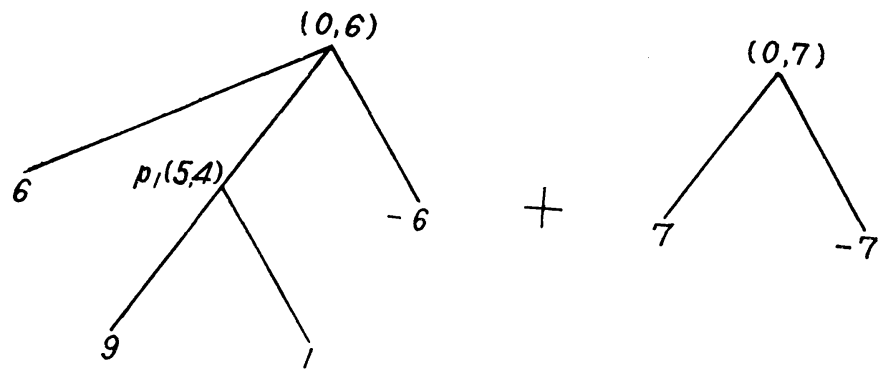

Figure 9

\section{REFERENCES}

1. John Milnor, Sums of positional games, Contributions to the theory of games, Vol. II, p. 291-301, Princeton, 1953.

2. Pólya-Szegö, Aufgaben und Lehrsätze aus der Analysis, Bd. 1, Berlin, 1925.

UNIVERSITY OF STOCKHOLM

SWEDEN 



\section{PACIFIC JOURNAL OF MATHEMATICS}

\section{EDITORS}

\section{David Gilbarg}

Stanford University

Stanford, California

R. A. Beaumont

University of Washington

Seattle 5 , Washington
A. L. Whiteman

University of Southern California

Los Angeles 7, California

L. J. PAIGE

University of California

Los Angeles 24, California

\section{ASSOCIATE EDITORS}
E. F. BECKENBACH
C. E. BURGESS
E. HEWITT
A. HORN

\author{
V. GANAPATHY IYER \\ R. D. JAMES \\ M. S. KNEBELMAN \\ L. NACHBIN
}
I. NIVEN
E. G. STRAUS
T. G. OSTROM
H. L. ROYDEN
G. SZEKERES
M. M. SCHIFFER
F. WOLF
K. YOSIDA

\section{SUPPORTING INSTITUTIONS}

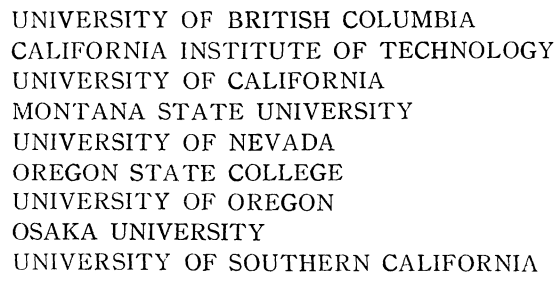

UNIVERSITY OF BRITISH COLUMBIA CALIFORNIA INSTITUTE OF TECHNOLOGY UNIVERSITY OF CALIFORNIA MONTANA STATE UNIVERSITY UNIVERSITY OF NEVADA OREGON STATE COLLEGE UNIVERSITY OF OREGON OSAKA UNIVERSITY UNIVERSITY OF SOUTHERN CALIFORNIA

STANFORD UNIVERSITY

UNIVERSITY OF TOKYO

UNIVERSITY OF UTAH

WASHINGTON STATE COLLEGE

UNIVERSITY OF WASHINGTON

AMERICAN MATHEMATICAL SOCIETY CALIFORNIA RESEARCH CORPORATION HUGHES AIRCRAFT COMPANY SPACE TECHNOLOGY LABORATORIES

Printed in Japan by Kokusai Bunken Insatsusha (International Academic Printing Co., Ltd.), Tokyo, Japan 


\section{Pacific Journal of Mathematics}

\section{Vol. 9, No. 1 \\ May, 1959}

Julius Rubin Blum and Murray Rosenblatt, On the structure of infinitely

divisible distributions . ............................. 1

Robert Geroge Buschman, Asymptotic expressions for

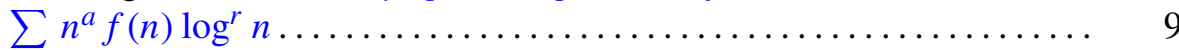

Eckford Cohen, A class of residue systems $(\bmod r)$ and related arithmetical

functions. I. A generalization of Möbius inversion .............. 13

Paul F. Conrad, Non-abelian ordered groups ................... 25

Richard Henry Crowell, On the van Kampen theorem............... 43

Irving Leonard Glicksberg, Convolution semigroups of measures ........ 51

Seymour Goldberg, Linear operators and their conjugates ............ 69

Olof Hanner, Mean play of sums of positional games .............. 81

Erhard Heinz, On one-to-one harmonic mappings ................ 101

John Rolfe Isbell, On finite-dimensional uniform spaces . . ........... 107

Erwin Kreyszig and John Todd, On the radius of univalence of the function

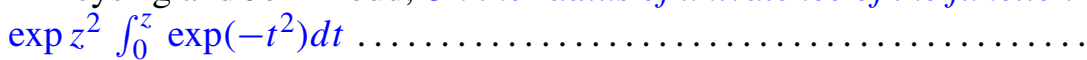

Roger Conant Lyndon, An interpolation theorem in the predicate

calculus......................................... 129

Roger Conant Lyndon, Properties preserved under homomorphism ........ 143

Roger Conant Lyndon, Properties preserved in subdirect products ....... 155

Robert Osserman, A lemma on analytic curves ................ 165

R. S. Phillips, On a theorem due to Sz.-Nagy..................... 169

Richard Scott Pierce, A generalization of atomic Boolean algebras ....... 175

J. B. Roberts, Analytic continuation of meromorphic functions in valued fields................................. 183

Walter Rudin, Idempotent measures on Abelian groups ................ 195

M. Schiffer, Fredholm eigen values of multiply-connected domains ........ 211

V. N. Singh, A note on the computation of Alder's polynomials ......... 271

Maurice Sion, On integration of 1-forms ...................... 277

Elbert A. Walker, Subdirect sums and infinite Abelian groups........... 287

John W. Woll, Homogeneous stochastic processes . .................. 293 